\title{
Formation trinationale Franco-Suisse-Allemande « Information Communication Systems » à l’Université de Haute Alsace
}

\author{
Alain Dieterlen*, Hubert Kihl**, Bernard Keltz* \\ * IUT de Mulhouse, 61 rue Albert Camus 68093 Mulhouse cedex \\ ** Faculté des Sciences et Techniques, 4 rue des Frères Lumière 68093 Mulhouse cedex
}

\begin{abstract}
RESUME : La formation trinationale «Information Communication Systems » a pour objectif la formation de Licence (Bachelor) dans le domaine des sciences pour l’ingénieur en électronique, automatique et informatique industrielle. Ce parcours est réalisé entre les trois partenaires de la Fachhochschule Nordwestschweiz en Suisse, la Hochschule de Furtwangen en Allemagne et l’Université de Haute Alsace à Mulhouse. Les étudiants provenant des baccalauréats scientifiques S ou STI, s'inscrivent dans ce cursus sur 3 années dont deux effectuées dans les universités partenaires et obtiennent les diplômes des trois pays. Le diplôme est validé lors du septième semestre par un stage de fin d'études dans une entreprise du domaine. La formation ICS fait partie d'un ensemble de formations transfrontalières proposées par l’Université de Haute Alsace. Son modèle de fonctionnement proche du LMD, fourni une souplesse de fonctionnement et permet son adaptation à d’autres domaines pour un coût de fonctionnement réduit.
\end{abstract}

Mots clés : Information Communication Systems, transfrontalier, interculturalité, diplômes reconnus, stages pratiques.

\section{INTRODUCTION.}

Fort d'une expérience de plus de 14 ans dans les relations transfrontalières avec nos voisins allemands et/ou suisses, l'Université de Haute Alsace (UHA) dispose d'une offre de formation dans les divers domaines de l'enseignement. Idéalement située au cœur de la « Régio » dans la « Vallée du Rhin Supérieur », l’UHA a mis en place des formations transfrontalières dans les IUT, les Facultés et les écoles d’ingénieurs. Ces relations viennent en complément à ce que propose la Confédération Européenne des Universités du Rhin Supérieur (Eucor) fondée en 1989 entre les universités de Fribourg en Brisgau, Bâle, Strasbourg, Kralsruhe et Mulhouse. Ce réseau Eucor favorise les échanges d'étudiants entre les universités en leur permettant de bénéficier des services des autres universités, et facilite la mise en place de coopérations dans le domaine de la recherche de la culture et du sport.

Les formations bi et tri-nationales répondent la plupart du temps à un besoin industriel fort avec un aspect d'interculturalité prépondérant dans des domaines très variés. Dans ce cadre, le domaine des sciences et technologies comporte 3 formations, la Mécatronique, Régio Chimica et notre formation ICS pour « Information Communication Systems ».

\section{HISTORIQUE}

Le premier projet de formation trinationale fut lancé en 1989 sous l'initiative de Georg H. Endress président du groupe Endress \& Hauser. Ce groupe fut créé en 1953 à Lörrach à la jonction des frontières allemandes, suisses et françaises, dans le but de fournir des appareils de mesures et des solutions pour les procédés industriels. Ce n'est qu'en 1997, que la première filière trinationale en Mécatronique vue le jour, elle combine la mécanique, l'électronique, l'informatique et le management de projets techniques. Cette formation originale d'ingénieurs, sur initialement 8 semestres, avait pour partenaire en Suisse la Fachhochschule Nordwestschweiz (FHNW) à Muttenz et la Berufsakademie à Lörrach (BA) en Allemagne. Suite à la mise en place du LMD, l'organisation de la formation à été ramenée au niveau Licence (Bachelor) en 6 semestres avec un $7^{\text {ième }}$ semestre de stage particulièrement important pour les partenaires suisses et allemands. Une particularité de cette formation est que les étudiants allemands sont tous sous contrat avec une entreprise, condition sine qua non pour l'inscription à la BA.

Dès le début, cette filière comportait un groupe de 30 étudiants, théoriquement 10 de chaque pays, qui pendant les 8 semestres (puis 7) formait une promotion qui suivait les cours alternativement dans les trois pays, suivant un calendrier semestriel entrecoupé de périodes de stages industriels. Les enseignants comme les étudiants sont amenés à se déplacer dans les deux autres pays, ce qui favorise l'interculturalité, l'un des objectifs majeur visé par ces formations internationales, qui montre l'apport de la mobilité transfrontalière dans des contextes éducatifs [1].

Dans le même esprit, notre formation trinationale, est définie autour d'objectifs disciplinaires dans le domaine des sciences pour l'ingénieur, plus précisément en électronique, automatique et informatique industrielle. Depuis 2003, suite à une première expérience infructueuse de mise en place d'une formation basée sur le modèle de la Mécatronique, nous travaillons avec la Fachhochschule Nordwestschweiz en Suisse et la Hochschule de Furtwangen en Allemagne sur le développement d'un parcours de Licence : « Information Communication Systems ».

En septembre 2007 à l'UHA, nous avons ouvert la formation aux étudiants français, pendant ce temps les collègues allemands engageaient la demande 
d'accréditation. L'adage de notre formation, décrite dans la suite :

Dépassez vos Frontières Sprengen Sie ihre Grenzen Etudiez dans trois pays studieren Sie in drei Ländern

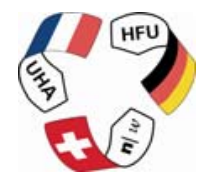

\section{INFORMATION COMMUNICATION SYS- TEMES}

Dans le domaine des sciences pour l'ingénieur, le cursus ICS s'articule autours des disciplines des systèmes électroniques, des systèmes informatisés et du management. Le partenaire Suisse est la Fachhochschule Nordwestschweiz dans le canton d'Argovie et le partenaire Allemand est la Hochschule de Furtwangen située en Forêt noire. La distance est d'une centaine de kilomètres entre chacun des trois lieux.

A l'origine de cette création deux constats, d'une part un trop faible taux de renouvellement des techniciens et ingénieurs dans le domaine scientifique qui en Allemagne se résume par un nouvel élément formé pour deux départ à la retraite, et d'autre part un tissus industriel entre les trois pays qui ne coopère que trop peu en raison de la barrière linguistique. Ces motivations sont en adéquation avec le bilan du livre blanc sur le dialogue interculturel réalisé en 2008 [2].

\subsection{Objectifs}

Outre les objectifs scientifiques dans le cadre de l'EEA en électronique, automatique et informatique industrielle, la notion de gestion de projet et de management est une compétence forte visée dans la formation ICS. Un second objectif particulièrement important est la notion d'interculturalité qui est vécue tout au long de la formation durant laquelle tous les étudiants vont passer au moins 2 semestres dans chacun des trois pays partenaires. La mobilité des étudiants nécessitera et développera une forte capacité d'adaptation sur des universités voisines, mais très différentes sur bien d'autres aspects. Cette interculturalité passe par la maitrise de la langue étrangère, pour les Français l'Allemand.

Le dernier objectif et non le moindre est l'insertion professionnelle à l'International des étudiants ayant suivi cette formation. La poursuite d'études en Master est envisagée pour ces étudiants particulièrement motivés, mais le stage de fin d'études d'un semestre est un premier pas important vers le monde industriel.

\subsection{Présentation pédagogique}

Le parcours de cette formation est basé sur le processus de Sorbonne-Bologne du premier cycle d'études avec l'obtention de 180 crédits ECTS théoriques, additionnés de 30 crédits liés au stage pratique réalisé lors d'un $7^{\text {ième }}$ semestre. Les étudiants obtiennent les diplômes des trois pays avec un supplément au diplôme récapitulant le parcours individuel de chaque récipiendaire.
La formation est décomposée en 4 modules et chaque étudiant est suivi dans chaque pays par un tuteur.

\subsubsection{Structure des modules}

Pour harmoniser le contenu de la formation et la rendre plus lisible par les étudiants, nous avons décomposé celle-ci en 4 modules décrits dans la figure $n^{\circ} 1$.

Un module de méthodologie (fig. $\mathrm{n}^{\circ} 1$ en vert), ayant pour objectif la formation au travail scientifique autour des projets et la rédaction du mémoire de stage, ce module sera au final de 30 crédits ECTS.

Un module de spécialisation (fig. $\mathrm{n}^{\circ} 1$ en bleu), dans lequel nous retrouvons les enseignements scientifiques de la formation avec des enseignements scientifiques de bases (mathématiques et physique), des enseignements d'électronique au sens large, des enseignements d'informatique et des systèmes d'informations. Ce module comportera en totalité un minimum de 102 crédits, qui pourront être complétés au choix.

Le module complémentaire regroupe les notions d'interculturalité, c'est à dire les langues indispensables à la formation pour un minimum de 14 crédits, des crédits de culture générale (minimum 10) et de spécialisation pouvant, par exemple, inclure les notions de management de projet par exemple. Ce module est important lors de la première année, ou les étudiants doivent se perfectionner dans la langue étrangère, allemand pour les français et français pour les suisses et allemands.

Le dernier module concerne la partie pratique du stage de fin d'étude au septième semestre, ce stage pratique peut se dérouler dans l'un des trois pays ou à l'international. Cette souplesse laisse un choix qui dans la réalité se concrétise jusqu’à présent par des stages effectuée dans un pays étranger. Le suivi du stage est confié à l'université dans laquelle l'étudiant a débuté sa formation, ceci résulte de raisons administratives d'encadrement de stage pour les français.

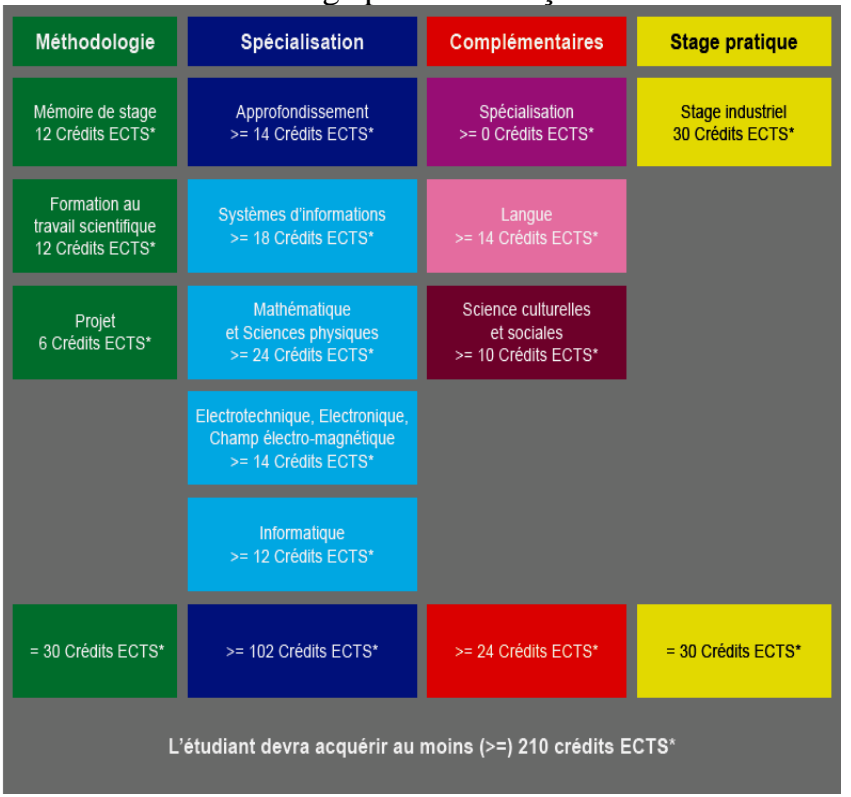


fig 1 : Présentation des modules et de leur contenus spécifiant la formation ICS.

La somme en ECTS des modules doit être de 210 crédits, sachant que les modules de Méthodologie et du stage pratique sont de 30 crédits chacun, la somme des deux autres modules sera donc de 150 ECTS. Le minimum fixé est de 126 crédits, chaque étudiant pourra donc choisir pour compléter et personnaliser son parcours 24 crédits durant les trois années. Ce choix est fait en concertation avec l'enseignant tuteur dans le pays d'accueil. Afin de garantir l'interculturalité, un minimum de 50 crédits ECTS devra être obtenu dans chacun des trois pays.

Enfin, la charge de travail considérée pour un crédit ECTS est de 30h : ceci inclut cours présentiel (Cours, Travaux dirigés et Pratiques) et travail personnel.

\subsubsection{Tuteur pédagogique}

Dans chaque université d'accueil, au moins un tuteur pédagogique suit l'évolution des étudiants. Son rôle principal est de les initier à un mode de fonctionnement différent d'un pays à l'autre et de les aider dans le choix des modules de leur parcours individualisé. Son impact est d'autant plus important que l'on avance dans la formation.

Pour l'aider dans sa démarche, le directoire (voir § 3.3.1.) de la formation a établi un tableau de correspondance entre les différents cours et leur poids en crédits ECTS. Cet important travail nécessite de la part des personnes impliquées une vraie collaboration. L'exercice est très délicat, il faut mesurer les niveaux différents de difficultés sur trois années pour éviter des redondances dans la formation. Ce travail n'est d'ailleurs jamais totalement abouti et nécessite des ajustements réguliers.

Mais ce travail collaboratif est particulièrement enrichissant pour les enseignants impliqués.

\subsubsection{Evaluation}

L'évaluation des étudiants durant les 6 semestres comporte de nombreuses différences, en premier la notation qui est sur une échelle de 0-20 en France de 1 à 6 en Allemagne et de 6 à 1 en Suisse ! Si ceci perturbe l'étudiant dans un premier temps, pour les enseignants il a également fallut mettre en place une échelle de notation commune basée sur les appréciations allant de « Insuffisant » à " Très bien ». Cette grille trouve son utilité lors de l'évaluation finale pour valider et donner une mention sur le diplôme.

Pour achever sa formation, l'étudiant devra valider les 180 crédits théoriques et les 30 crédits du stage pratique. Le directoire en réunion valide les parcours de chacun et transmet les résultats à chaque université partenaire pour l'obtention du grade de Licence ou de Bachelor. Un supplément au diplôme est établi pour chaque étudiant avec la mention «formation trinationale ICS ».

\subsection{Fonctionnement administratif}

\subsubsection{Partenariat}

Depuis le début de la collaboration en 2003, jusqu’à la mise en œuvre de la formation en 2007, et désormais pour le suivi des étudiants et l'évolution du contenu pédagogique ainsi que la communication, les trois partenaires se réunissent au minimum tous les 3 mois. Le consortium, appelé directoire, est constitué d'au moins de 2 enseignants de chaque université.

Dans un premier temps en septembre 2005, un accord de consortium fixant les objectifs avait été signé par les trois présidents des universités. Chaque partenaire s'engageant à développer le parcours dans son université avec ses propres moyens. Des demandes de soutiens conjoints sont déposées dans le but de favoriser la mobilité des étudiants et leur renforcement dans la langue étrangère. L'Université Franco-Allemande (UFA) peut venir en support de ce type de formation, mais ses moyens limités ne permettent pas de financer toutes les initiatives (dont la notre). Pour l'instant les moyens mis en œuvre pour la promotion de la formation ICS restent ceux de chaque université. Le coût de la formation est de ce fait particulièrement faible, essentiellement pour la communication et un peu de fonctionnement, les enseignements étant ceux existant dans les universités.

\subsubsection{Communication}

Si le point de départ de la formation émane d'une volonté de partenaires industriels (Endress \& Hauser), la promotion de ce type de formation est importante, le potentiel d'étudiants dans le domaine scientifique est malheureusement assez faible, et ceci de part et d'autre du Rhin. De plus, l'apprentissage de la langue est souvent un frein chez les étudiants visés, cette barrière est pourtant souvent assez facilement franchie et ceci grâce à l'immersion dans le pays.

Le lancement de la formation avait réuni le 4 juin 2007 plus de 70 participants, partenaires politiques et industriels, à la Konzerthaus de Freiburg (DE). La table ronde organisée a conforté la nécessité d'une interaction forte entre le monde industriel et le milieu universitaire.

Depuis cette date, nous participons au forum francoallemand à Strasbourg au mois de novembre qui regroupe les acteurs des formations post-bac entre la France, l'Allemagne, l'Autriche et la Suisse.

D’autre part, nous profitons d'évènements tels que « la fête de la science " pour mettre en avant la formation avec l'implication des collègues suisses et allemands. Ces occasions sont également des moments ou les étudiants se retrouvent sur des projets communs, comme par exemple des concours de robotique.

Parfois cette collaboration s'étend avec des échanges plus importants. Par exemple, l'exposition FETTU sur les photos de notre univers, montée à l'UHA, avait séjourné pendant un mois à la Hochschule de Furtwangen. 
Bien entendu le vecteur de communication qu'est Internet est utilisé, le nom du site hébergeant la formation avait été déposé dès la création de la formation: http://www.ics-trinational.eu/

\subsubsection{Habilitation - Accréditation}

Ce cursus pour être diplômant est validé par les différents organismes propres à chaque pays.

Les allemands passent par une accréditation réalisée par des organismes extérieurs, l'évaluation de la qualité de la formation porte aussi sur l'évaluation de la qualité des partenaires. Une visite d'experts dans les trois universités a eu lieu en mars 2008, l'accréditation a été obtenue au mois de juin de la même année.

A l'UHA cette formation s'appuie sur des formations existantes à l'IUT et la Faculté des Sciences et Techniques. Plus précisément, les deux première années correspondent au DUT GEii et deux derniers semestres (5 et 6) à la licence EEA. Ce parcours trinational ICS dans les deux composantes de l'UHA ne nécessite donc pas de création. Nous avons néanmoins obtenu la validation de ce parcours par le CEVU de notre Université, il apparait dans l'offre de licence EEA du quadriennal. Cette souplesse nous avait permis de débuter le cursus une année avant les collègues allemands et suisses.

\subsubsection{Inscription}

Afin d'harmoniser les frais des étudiants durant leur parcours, sachant que le coût de l'inscription en France est d'un tiers par rapport à celui de l'inscription en Suisse et en Allemagne, il a été décidé que chaque étudiant paye les droits d'inscription dans l'université d'accueil. De ce fait, au final un étudiant français, suisse ou allemand investit la même somme pour ses trois années. Le problème de la couverture sociale des Français est résolu par une inscription administrative simplifiée sans frais.

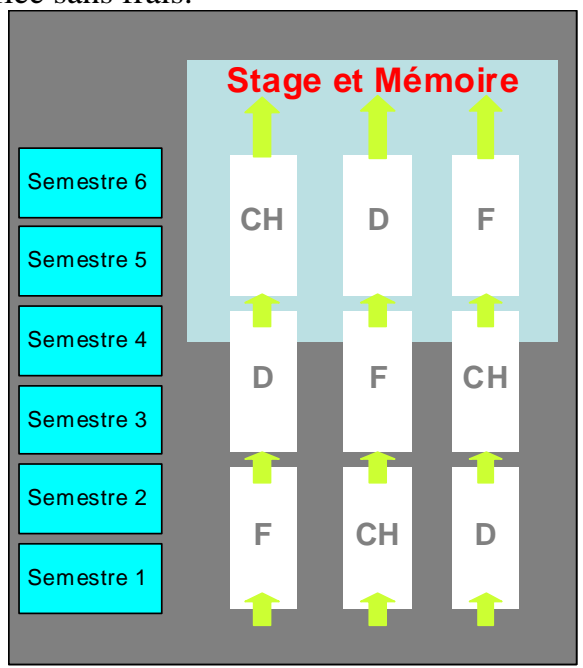

fig 2 : Parcours des étudiants Français (F) Suisses $(\mathrm{CH})$ et Allemands $(\mathrm{D})$ dans les trois pays du premiers au sixième semestre.
Pratiquement, les étudiants débutent leurs parcours dans leur pays d'origine, la figure $n^{\circ} 2$ montre l'ordre de rotation des étudiants durant les 6 semestres dans les trois pays. Cette rotation tient compte de l'effort linguistique à fournir par chacun, les français bénéficiant d'un avantage, puisqu'ils passeront deux tiers de leur formation dans un pays germanophone. L'avantage pour l'étudiant de passer sa première année de formation dans sa langue natale est de lui permettre d'améliorer son niveau en langue sans répercussion sur les bases scientifiques nécessaires pour la suite du parcours.

Actuellement la formation ICS concerne 12 étudiants français et 6 étudiants allemands et suisses. Les 12 étudiants français se répartissent sur les 7 semestres, les 4 premiers sont actuellement dans le dernier semestre en stage pratique dans l'industrie.

\section{DEVENIR DES ETUDIANTS}

Les premiers étudiants sortent de la filière, ils sont actuellement en stage de fin d'étude et devraient être diplômés en juin 2011. Certains pour cette période bénéficient d'un contrat à durée déterminée d'un an. Il est probable que cette première vraie expérience professionnelle leur ouvre des portes dans le monde professionnel. Cependant depuis le début de la création, nous sommes convaincus par le potentiel de ces étudiants à poursuivre des études par un Master ou dans une école d’ingénieur.

La principale difficulté dans la poursuite d'étude est le timing dicté par le septième semestre de stage. En France, ce décalage est problématique pour les étudiants qui sont décalés sur le rythme des semestres. Pour les premiers, le choix a été de prendre une année pour effectuer le semestre de stage. Une solution possible est de débuter cette phase pratique en fin de seconde année, et de compléter cette période en fin de sixième semestre, ceci permettra de débuter un Master ou d'intégrer une école d'ingénieur.

Actuellement, nous projetons de créer un master International pour compléter cette formation, un nouveau partenaire serait la John Moores Université à Liverpool.

\section{CONCLUSION}

Cette formation trinationale répond à une volonté régionale de développer les relations et partenariats économiques entre les trois pays. Suite à un recul important ces dernières années (plus de 10000) des travailleurs frontaliers français en Suisse, de nombreux efforts sont consentis par le conseil général du HautRhin. Ce Parcours est en phase avec la relance de la langue et de la culture régionale en Alsace.

Actuellement, après 3 années universitaire de fonctionnement, le flux d'étudiant reste faible, un objectif raisonnable serait d'accueillir jusqu'à 6 étudiants par an et par pays. Pour les Français ceci est potentiellement envisageable grâce à un nombre croissant de bachelier venant de sections scientifiques Abi-Bac (Abitur et 
Baccalauréat) ou renforcées en Allemand, la barrière de la langue n'étant plus un obstacle. Cependant économiquement, le potentiel d'emploi reste bien plus fort dans notre domaine en Allemagne et en Suisse, ce qui rend le recrutement français plus important dans ces formations scientifiques transfrontalières. C'est l'aspect International et l'ouverture de Master qui sera attractif pour les étudiants des autres pays.

Les étudiants des ces formations ont développé un fort potentiel d'adaptabilité, des compétences interculturelle et scientifiques importantes, et sont de très bons ambassadeurs de la qualité des formations scientifiques.

La collaboration avec les collègues ne se concentre pas seulement sur les aspects de l'enseignement, nous avons depuis deux ans mis en place une convention de partenariat de recherche avec l'Université de Furtwangen dans le domaine du Traitement d'Image commun au laboratoire MIPS de l'UHA.

\section{Bibliographie}

[1] Thiéblemont-Dollet S. " L'interculturalité dans tous ses états ", Nancy, Presses Universitaires de Nancy.

[2] Conseil de l'Europe, Strasbourg, Livre blanc sur le dialogue interculturel, COE, 2008,

http://www.coe.int/t/dg4/intercultural/Source/White\%20

Paper_final_revised_FR.pdf 